\title{
Nanophotonics
}

SPIEDigitalLibrary.org/jnp

\section{Nanofabrication Handbook}

\author{
Raúl J. Martín-Palma
}




\section{BOOK REVIEWS}

\section{Nanofabrication Handbook}

Stefano Cabrini and Satoshi Kawata, Eds., 546 pages, 463 illustrations, ISBN: 978-1-42009052-9 (hardback) and 978-1-4200-9053-6 (eBook), CRC Press, Boca Raton (2012), £82.00 (hardback).

Reviewed by Raúl J. Martín-Palma, Departamento de Física Aplicada, Universidad Autónoma de Madrid, 28049 Cantoblanco, Madrid, Spain, rauljose.martin@uam.es

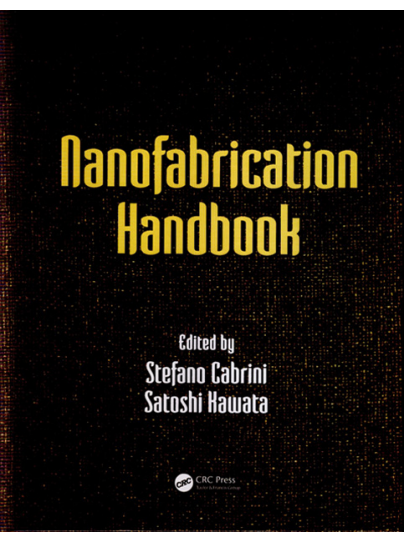

Nanofabrication Handbook is a contributed book edited by S. Cabrini (director, Molecular Foundry Nanofabrication Facility, Lawrence Berkeley National Laboratory, USA) and Satoshi Kawata (professor, Department of Applied Physics, Osaka University, Japan) and published by CRC Press.

First and foremost, it has to be pointed out that the main objective of the editors, i.e., to develop a single source providing a total vision of nanofabrication, has been fulfilled. There are many good books partly covering this huge field, but Nanofabrication Handbook covers both traditional and new lithographic techniques, as well as the most significant current applications.

However, maybe the most striking issue, realized when holding this book for the first time, is that the format chosen by the publisher does not seem to be the most appropriate one for a publication named "handbook." Given its dimensions ( 8.5 in $\times 10.8$ in $\times 1.4$ in) and weight (around $3.7 \mathrm{lb}$ ), the hardback version of the book is far from being a pocket-sized book, although the book fulfills the other meaning of the term. It provides a ready reference for current nanofabrication techniques, at both the micro- and nanoscales, and includes many applications. Of course, this apparent contradiction vanishes in the case of the ebook version.

As the editors state in their preface, nanofabrication is "a field that is constantly broadening its definition." However, Cabrini and Kawata have been able to put together a fine collection of chapters covering the state of the art in a rapidly evolving field. There is no doubt that all contributing authors are experts in their respective fields.

Nanofabrication Handbook comprises 22 chapters divided into three main parts: "Standard Lithography," "New Lithographic Techniques," and "Nanofabrication Applications." Clearly then, this book goes beyond the description of most nanofabrication techniques to present several practical applications. This is a positive aspect of this book, particularly since a group of nanofabrication techniques outside the "traditional" ones are discussed. Also, on the bright side, the profuse use of drawings, illustrations, and pictures will help the reader visualize what is explained and discussed throughout the text. Additionally, 24 color figures are included.

The overall level of this handbook makes it appropriate both for researchers/scientists/engineers wishing to learn the fundamentals of lithographic techniques and for researchers/scientists/ engineers with some experience in the field willing to learn advanced techniques and current practical applications.

Although all chapters are written by experts in their respective fields from a large number of institutions and research centers spread all over the world, the level and coverage of the different techniques and applications are not homogeneous. Also, the structure of each chapter is somewhat different. For instance, in Chapter 2 ("Electron Beam Lithography") boxes explaining physical effects, principles, and other helpful information are profusely used, but no boxes 
at all are present in most other chapters. As such, some chapters can be said to show an accentuated academic style while others more resemble a review paper.

Maybe, Chapter 19 ("Patterning Magnetic Nanostructures with Ions") should have been placed either in Part I ("Standard Lithography") or in Part II ("New Lithographic Techniques"). Or it could have even been merged with Chapter 3 ("Nanofabrication with Focused Ion Beams"). As is usually the case in contributed books, there is some partial overlap among chapters. For instance, there is an evident overlap between the topics discussed in Chapters 18 ("Fabrication of Nanophotonic Structures") and 20 ("Plasmonic Metamaterials"). Perhaps, these two chapters should have been fused into one. Quite likely, partial overlaps between some of the chapters will be unimportant to most readers.

Among the applications presented and discussed in Part III, the following are of intense research activity: Micro- and nanoelectromechanical systems (MEMS and NEMS), microand nanofluidics, nanophotonic structures (including metamaterials and plasmonics), nanomagnetic devices, nanoelectronics, and biological applications. As such, most applications of current interest are well covered. But insight into future uses and applications of nanofabrication techniques should have been provided at the end of the book, rather than in many chapters separately.

In summary, this handbook provides a very good overview of the state of the art in the field of nanofabrication using micro- and nanolithography. At the same time, it includes most of the current applications of these fabrication techniques. In this regard, both the fundamentals and practical applications related to nanofabrication by lithographic techniques are well covered. Moreover, every chapter of Nanofabrication Handbook includes a considerable list of references. Additional homogeneity and cross-referencing between the individual chapters would have been desirable, although probably this will not represent a major drawback to most readers who will probably just read a few individual chapters depending on their research interests. 